\title{
Four-Dimensional Nilpotent Diassociative Algebras
}

\section{Basri W, Rakhimov IS* and Rikhsiboev IM}

Universiti Putra Malaysia, Serdang, Selangor D.E., Malaysia

Universiti Kuala Lumpur MITEC, Johor Bahru, Johor D. T., Malaysia

\begin{abstract}
The paper is devoted to structural properties of diassociative algebras. We introduce the notions of nilpotency, solvability of the diassociative algebras and study their properties. The list of all possible nilpotent diassociative algebra structures on four-dimensional complex vector spaces is given.
\end{abstract}

Keywords: Associative algebra; Diassociative algebra; Isomorphism; Nilpotency; Solvability

\section{Introduction}

In 1993, Loday introduced the notion of Leibniz algebra, that is a generalization of Lie algebra, where the skew-symmetricity of the bracket is dropped and the Jacobi identity is replaced by the Leibniz identity (the identity has been called Leibniz identity by Loday due to its similarity to Leibniz rule, this is the reason for the class to be called by the name of Leibniz). Loday also showed that the link between Lie and associative algebras can be extended to analogous link between Leibniz algebras and a so-called associative dialgebras which are a generalization of associative algebras possessing two composition laws. Loday showed that if $D=(V, \vdash,-)$ is a diassociative algebra structure on a vector space $V$ then the Leibniz algebra structure on $V$ is defined by the bracket $[x, y]:=x \dashv y-y \vdash x$. Conversely, the universal enveloping algebra of Leibniz algebra has the structure of diassociative algebra. Loday has given some examples to motivate the study of these classes of algebras. On the structure of algebras from these classes not so much is known. Researchers (Loday, Pirashvili and others) mainly focused on their (co)homological problems.

The study of structural properties of Leibniz algebras has been initiated by Ayupov and Omirov [1]. Casas gave the list of isomorphism classes of three-dimensional complex Leibniz algebras [2] (twodimensional case has been given by Loday himself). The list of nilpotent Leibniz algebras in dimension four has been obtained in [3]. In higher dimensions there are classification results with stronger conditions.

A diassociative algebra is a vector space with two bilinear binary associative operations $\vdash,-$, satisfying certain conditions [4]. Associative algebras are particular case of the diassociative algebras when the two operations coincide. The main motivation of Loday to introduce this class of algebras was the search of an "obstruction" to the periodicity in algebraic K-theory. Besides this purely algebraic motivation some their relations with classical and non-commutative geometry, and physics have been recently discovered.

The classification problem of algebras is one of the important problems of modern algebra. The problem has been successfully solved for semisimple parts of many classes of finite-dimensional algebras. However, the complete classification of solvable and nilpotent parts is still unsolved. Particularly, for Lie, Leibniz and associative algebras the solution to the problem in low-dimensional cases has been given with some conditions.

One of the approaches is to use the conditions on structure constants due to axioms of the class of algebras considered. As for diassociative algebras none of the above mentioned parts are studied. In this paper we are interested in description of diassociative algebra structures on low-dimensional complex vector spaces. We construct a diassociative algebra structure on a vector space as a combination of two associative algebras. Accordingly, we need complete lists of associative algebras (both, unital and non unital) in the dimensions considered. The lists can be found in $[5,6]$.

We remind that the classification problems of low-dimensional complex Lie and Leibniz algebras have been considered in $[1,3,7,8]$. In this paper we give the list of isomorphism classes of four-dimensional complex nilpotent diassociative algebras. We restrict our discussion to four-dimensional nilpotent case since in lower dimensions the classifications have already been given in $[9,10]$.

The organization of the paper is as follows. It is split into four sections including the introduction. In Section 2 we give necessary definitions needed and present the lists of complex diassociative algebras in dimensions two and three from $[9,10]$. The main results of this paper are in Sections 3 and 4. In Section 3 we propose the notions of nilpotency and solvability for diassociative algebras and give criteria of nilpotency and solvability of dialgebras. In Section 4 the complete list of isomorphism classes of four-dimensional nilpotent complex diassociative algebras are given.

\section{Loday Algebras}

Around 1990, Loday introduced several new classes of algebras called Leibniz algebra, diassociative algebra, dendriform algebra and Zinbiel algebra $[4,11]$. He also has given a categorical diagram on links between these and classical algebras. In this section we briefly review definitions and remind the classification results of Loday algebras.

Definition 1: A Leibniz algebra is a vector space L equipped with a binary operation, called Leibniz bracket, [•, •] : L $\times L \rightarrow L$ which satisfies the Leibniz identity

$[[x, y], z]=[[x, z], y]+[x,[y, z]]$, for all $x, y, z \in L$.

Obviously, if the bracket is skew-symmetric, then $L$ is a Lie algebra .

It is well known that an associative algebra $(A, \bullet)$ gives rise to a Lie

${ }^{*}$ Corresponding author: Rakhimov IS, Universiti Putra Malaysia Serdang Selangor D.E., Malaysia, Tel: +60389466831; E-mail: risamiddin@gmail.com

Received August 28, 2014; Accepted April 27, 2015; Published May 04, 2015

Citation: Basri W, Rakhimov IS, Rikhsiboev IM (2015) Four-Dimensional Nilpotent Diassociative Algebras. J Generalized Lie Theory Appl 9: 218. doi: 10.4172/17364337.1000218

Copyright: $\odot 2015$ Basri W, et al. This is an open-access article distributed under the terms of the Creative Commons Attribution License, which permits unrestricted use, distribution, and reproduction in any medium, provided the original author and source are credited. 
algebra by $[a, b]=a \bullet b-b \bullet a$. There is a generalization of associative algebra which plays the same role to Leibniz algebra as associative algebra to Lie algebra in the above link. This generalization is called associative dialgebra. The definition of the associative dialgebra is as follows.

Definition 2: Associative dialgebra (the term diassociative algebra also is used) $D$ is an algebra equipped with two bilinear binary associative operations, $\dashv$ and $\vdash$, called left and right products, respectively, satisfying the following axioms:

$$
\begin{aligned}
& (x \dashv y) \dashv z=x \dashv(y \vdash z), \\
& (x \vdash y) \dashv z=x \vdash(y \dashv z), \\
& (x \dashv y) \vdash z=x \vdash(y \vdash z),
\end{aligned}
$$

for all $x, y, z \in D$.

Let $(D, \dashv, \vdash$, , ) be a diassociative algebra structure on a vector space $V$ then it is immediate to check that the bracket $[x, y]:=x \dashv y-y \vdash x$ satisfies the Leibniz identity (1) and defines a Leibniz algebra structure on $V$.

Some examples and applications of diassociative algebras are given in [12-14].

We remind the definitions of a few more classes of algebras also introduced by Loday and (co)homologically closely related to the above mentioned two classes of algebras in order to complete Loday's categorical diagram. One of these classes is called the class of dendriform algebras.

Definition 3: Dendriform algebra $E$ is an algebra with two binary operations

$$
>: E \times E \rightarrow E, \prec: E \times E \rightarrow E
$$

satisfying the axioms:

$$
\begin{gathered}
(x \prec y) \prec z=x \prec(y \prec z)+x<(y>z), \\
(x>y) \prec z=x>(y \prec z), \\
(x \prec y)>z+(x>y)>z=x>(y>z),
\end{gathered}
$$

for all $x, y, z \in E$.

The papers [4,15-17] are devoted to the dendriform algebras and their relations.

Another class of algebras introduced by Loday is a class called Zinbiel algebras. The definition of the Zinbiel algebra is as follows.

Definition 4: Zinbiel algebra $R$ is an algebra with a binary operation $\bullet: R \times R \rightarrow R$, satisfying the condition:

$(x \cdot y) \cdot z=x \cdot(y \cdot z)+x \bullet(z \bullet y)$, for all $x, y, z \in R$.

The essential progress on Zinbiel algebras has been made by A. Dzhumadildaev et al. in [18], where the authors proved that any finite-dimensional Zinbiel algebra over an algebraic closed field is nilpotent, nil and solvable. A few years before Omirov [19] have given the classification of two-dimensional complex Zinbiel algebras. Most studied class among the classes of algebras introduced by Loday is the class of Leibniz algebras. Complete classifications up to dimension three are given [2], nilpotent case in dimension four can be found in [3], the solvable Leibniz algebras have been studied in [20,21]. In higher dimensions there are classifications of subclasses of nilpotent part (filiform Leibniz algebras) [22-24].
The results intertwining Loday algebras are best expressed in the framework of algebraic operads. The notion of diassociative algebra defines an algebraic operad Dias, which is binary and quadratic. According to the theory of Ginzburg and Kapranov there is a welldefined "dual operad" Dias!. Loday has showed that this is exactly the operad Dend of the dendriform algebras, in other words dual diassociative algebra is nothing but a dendriform algebra. The similar duality can be established between the algebraic operads Leib defined by the notion of Leibniz algebra and the algebraic operads Zinb defined by the notion of Zinbiel algebra. The categories of algebras over these operads assemble into a commutative diagram of functors which reflects the Koszul duality [4]

The classification of these new classes of algebras is of great interest. In this paper we deal with the classification problem of diassociative algebras.

Now and what it follows we use the following notations: $A s^{q}{ }_{n}$ and Dias $_{n}{ }_{n}$ stand for $q$ th associative and $q$ th diassociative algebra structures in $n$-dimensional vector space, respectively. All algebras and vector spaces are assumed to be finite-dimensional and over the field of complex numbers $\mathbb{C}$, unless is stated otherwise.

There is the following naive approach for classifying of algebra structures on a vector space which often is being used up to now. It runs as follows. Once one fixes a basis of the vector space then according to the identities which the algebra satisfies we get a system of equations with respect to the structure constants of the algebra on this basis. Solving this system of equations we get a redundant, in general, list of algebras via the tables of multiplications. The second step is to make the obtained list irredundant. The irredundancy can be achieved by identifying those algebras which are obtained from others by a base change. This approach has been applied to get classifications of two and three-dimensional diassociative algebras over $\mathbb{C}$. The results are given by the next two theorems followed by a discussion on the structure constants of diassociative algebras.

Let $V$ be an $n$-dimensional vector space and $(D, \vdash,-1)$ be a diassociative algebra structure on $V$. Choose a basis $e_{1}, e_{2}, \ldots, e_{n}$ of $V$. A diassociative algebra structure on $V$ is determined by the set of structure constants $\alpha_{i j}^{k}$ and $\beta_{i p}^{q}$.

$$
e_{i} \vdash e_{j}=\sum_{k=1}^{n} \alpha_{i j}^{k} e_{k}, \quad e_{1} \dashv e_{p}=\sum_{t=1}^{n} \beta_{\mathrm{lp}}^{t} e_{t}, \text { for } 1 \leq i, j, 1, p \leq n .
$$

The diassociative algebra axioms give the following constrains for $\alpha_{i j}^{k}$ and $\beta_{i p}^{q}$ :

$\alpha_{i j}^{r} \alpha_{r k}^{s}=\alpha_{i r}^{s} \alpha_{j k}^{r}, \alpha_{i j}^{r} \alpha_{j k}^{r}=\alpha_{i j}^{r} \beta_{r k}^{q}, \alpha_{j k}^{r} \beta_{i r}^{s}=\alpha_{i j}^{k} \beta_{j k}^{r}, \alpha_{i j}^{r} \beta_{r k}^{s}=\beta_{i r}^{s} \beta_{j k}^{r}, \beta_{i j}^{r} \beta_{r k}^{s}=\beta_{i r}^{s} \beta_{j k}^{r}$

The classification result below for two-dimensional complex diassociative algebras is obtained by solving two-dimensional version of the system of equations above [9].

Theorem 2.1: Any diassociative algebra structure on twodimensional complex vector space is isomorphic to one of the following classes of algebras:

$$
\begin{aligned}
& { }_{\operatorname{Dias}_{2}}: e_{1} \dashv e_{1}=e_{1}, e_{1} \dashv e_{1}=e_{1}, e_{2} \dashv e_{1}=e_{2} ; \\
& \operatorname{Dias}_{2}^{2}: e_{1} \dashv e_{1}=e_{1}, e_{1} \dashv e_{2}=e_{2}, e_{1} \dashv e_{1}=e_{1} ; \\
& { }^{3}:(\alpha): e_{1} \vdash e_{1}=e_{2}, e_{1} \dashv e_{1}=\alpha e_{2}, \quad \alpha \in \mathbb{C} \backslash\{0\} ; \\
& { }^{4}: e_{1} \vdash e_{1}=e_{1}, e_{1} \dashv e_{2}=e_{2}, e_{1} \dashv e_{1}=e_{1}, e_{2} \dashv e_{1}=e_{2} .
\end{aligned}
$$


Here is the classification result in three-dimensional case obtained in [10].

Theorem 2.2: Any three-dimensional complex diassociative algebra can be included in one of the following isomorphism classes of diassociative algebras:

$$
\begin{aligned}
& { }_{\operatorname{Dias}_{3}}{ }^{1}: \quad e_{1} \dashv e_{2}=\mathrm{e}_{1}, \quad e_{2} \dashv e_{2}=e_{2}, \quad e_{3} \dashv e_{3}=e_{3} \text {, } \\
& e_{2} \vdash \mathrm{e}_{2}=e_{2}, \quad e_{3} \vdash e_{3}=e_{3} \text {; } \\
& \operatorname{Dias}_{3}^{2}: \quad e_{1} \dashv e_{2}=e_{1}, \quad e_{2} \dashv e_{2}=e_{2}, \quad e_{3} \dashv e_{3}=e_{3} \text {, } \\
& e_{2} \vdash e_{1}=e_{1}, \quad e_{2} \vdash e_{2}=e_{2}, \quad e_{3} \vdash e_{3}=e_{3} \text {; } \\
& \operatorname{Dias}^{3}{ }^{3}: \quad e_{1} \dashv e_{2}=e_{1}, \quad e_{2} \dashv e_{2}=e_{2}, \quad e_{3} \dashv e_{3}=e_{3} \text {, } \\
& e_{2} \vdash e_{2}=e_{2}, \quad e_{3} \vdash e_{1}=e_{1} \text {; } \\
& \operatorname{Dias}_{3}{ }^{4}: \quad e_{1} \dashv e_{3}=e_{2}, \quad e_{2} \dashv e_{3}=e_{2}, \quad e_{3} \dashv e_{3}=e_{3} \text {, } \\
& e_{3} \vdash e_{3}=e_{3} \text {; } \\
& \operatorname{Dias}_{3}^{5}: \quad e_{1} \dashv e_{3}=e_{2}, \quad e_{2} \dashv e_{3}=e_{2}, \quad e_{3} \dashv e_{3}=e_{3}, \\
& e_{3} \vdash e_{1}=e_{1}-e_{2}, e_{3} \vdash e_{3}=e_{3} \text {; } \\
& \text { Dias }_{3}^{6}: \quad e_{1} \dashv e_{3}=e_{2}, \quad e_{2} \dashv e_{3}=e_{2}, \quad e_{3} \dashv e_{3}=e_{3} \text {, } \\
& e_{3} \vdash e_{1}=e_{1}, \quad e_{3} \vdash e_{2}=e_{2}, \quad e_{3} \vdash e_{3}=e_{3} ; \\
& \operatorname{Dias}_{3}^{7}: \quad e_{1} \dashv e_{3}=e_{2}, \quad e_{2} \dashv e_{3}=e_{2}, \quad e_{3} \dashv e_{3}=e_{3}, \\
& e_{3} \vdash e_{1}=e_{2}, \quad e_{3} \vdash e_{2}=e_{2}, \quad e_{3} \vdash e_{3}=e_{3} ; \\
& \text { Dias }_{3}^{8}: \quad e_{1} \dashv e_{3}=e_{2}, \quad e_{2} \dashv e_{3}=e_{2}, \quad e_{3} \dashv e_{3}=e_{3} \text {, } \\
& e_{1} \vdash e_{3}=e_{2}, \quad e_{2} \vdash e_{3}=e_{2}, \quad e_{3} \vdash e_{1}=e_{1}-e_{2}, \\
& e_{3} \vdash e_{3}=e_{3} \text {; } \\
& \operatorname{Dias}_{3}^{9}: \quad e_{3} \dashv e_{1}=e_{2} \text {, } \\
& e_{3} \vdash e_{1}=e_{1} \text {, } \\
& e_{3} \dashv e_{2}=e_{2}, \\
& e_{3} \vdash e_{2}=e_{2} \text {, } \\
& \operatorname{Dias}_{3}^{10}: \quad e_{3} \dashv e_{1}=e_{1}, \\
& e_{3} \vdash e_{1}=e_{1} \text {, } \\
& e_{2} \dashv e_{3}=e_{2} \text {, } \\
& e_{3} \vdash e_{3}=e_{3} \text {; } \\
& \operatorname{Dias}_{3}^{11}: \quad e_{3} \dashv e_{1}=e_{1}, \\
& e_{3} \vdash e_{1}=e_{1} \text {, } \\
& e_{2} \dashv e_{3}=e_{2} \text {, } \\
& e_{3} \vdash e_{2}=e_{2} \text {, } \\
& e_{3} \dashv e_{3}=e_{3} \text {, } \\
& e_{3} \vdash e_{3}=e_{3} \text {; } \\
& e_{3} \dashv e_{3}=e_{3} \text {, } \\
& e_{3} \dashv e_{3}=e_{3} \text {, } \\
& \operatorname{Dias}_{3}^{12}: \quad e_{1} \dashv e_{3}=e_{1}, \\
& e_{2} \dashv e_{3}=e_{2} \text {, } \\
& e_{3} \vdash e_{3}=e_{3} \text {; } \\
& e_{3} \dashv e_{3}=e_{3} \text {, } \\
& e_{3} \vdash e_{3}=e_{3} \text {; } \\
& e_{1} \vdash e_{3}=e_{1} \text {, } \\
& e_{3} \dashv e_{1}=e_{1} \text {, } \\
& e_{3} \vdash e_{1}=e_{1} \text {, } \\
& \operatorname{Dias}_{3}^{13}: \quad e_{1} \dashv e_{3}=e_{1} \text {, } \\
& e_{2} \dashv e_{3}=e_{2} \text {, } \\
& e_{3} \dashv e_{1}=e_{1}, \\
& e_{3} \dashv e_{3}=e_{3} \text {, } \\
& e_{1} \vdash e_{3}=e_{1} \text {, } \\
& e_{3} \vdash e_{2}=e_{2} \text {, } \\
& e_{3} \vdash e_{3}=e_{3} \text {; } \\
& e_{3} \vdash e_{3}=e_{3} \text {; } \\
& e_{3} \vdash e_{3}=e_{3} \text {, } \\
& \operatorname{Dias}_{3}^{16}: \quad e_{1} \dashv e_{3}=e_{2} \text {, } \\
& e_{1} \vdash e_{3}=n e_{2} \text {, } \\
& e_{1} \dashv e_{1}=\alpha e_{2} \text {, } \\
& e_{3} \dashv e_{1}=k e_{2}, \\
& e_{3} \vdash e_{1}=p e_{2} \text {, } \\
& \operatorname{Dias}_{3}^{17}: e_{1} \dashv e_{2}=e_{2}, \quad e_{1} \dashv e_{2}=e_{3} \text {, } \\
& e_{1} \vdash e_{1}=e_{2}+e_{3}, \quad e_{1} \vdash e_{2}=e_{3} \text {, } \\
& \operatorname{Dias}_{3}^{18}: \quad e_{3} \dashv e_{3}=e_{3}, \quad e_{3} \vdash e_{1}=e_{2} \text {, } \\
& e_{3} \vdash e_{3}=e_{3} \text {. }
\end{aligned}
$$

$\forall \alpha, k, m, n, p, q \in \mathbb{C}$;

According to the theorem, among the nineteen isomorphism classes of three-dimensional diassociative algebras there are seventeen single representatives and two of the classes are represented by parametric family of algebras.

\section{On Nilpotency and Solvability of Diassociative Algebras}

In this section we give natural extensions of the concepts of nilpotency and solvability of algebras to diassociative algebras case. Two important criteria (Theorem 3.1 and Theorem 3.2) verifications of nilpotency and solvability of the diassociative algebras are given.

Let $M$ and $N$ be subsets of a diassociative algebra $D$. We define the following binary operation, denoted $\diamond$, over the subsets $M$ and $N$ of $D$ :

$$
M \diamond N:=M \vdash N+M \dashv N,
$$

where

$$
M \vdash N=\operatorname{Span}_{\mathrm{C}}\left\{d_{i} \vdash d_{j} \mid d_{i} \in M, d_{j} \in N\right\}
$$

and

$$
M \dashv N=\operatorname{Span}_{\mathbb{C}}\left\{d_{k} \dashv d_{s} \mid d_{k} \in M, d_{s} \in N\right\} .
$$

Obviously, if $M$ and $N$ are ideals so is $M \diamond N$. Let us consider the following series (central series) of ideals:

$$
\begin{aligned}
& \text { I. } D^{<1>}=D, D^{<k+1>}=D^{<k>} \diamond D, \\
& \text { II. } D^{\{1\}}=D, D^{\{k+1\}}=D \diamond D^{\{k\}}, \\
& \text { III. } D^{1}=D D^{k+1}=D^{1} \diamond D^{k}+D^{2} \diamond D^{k-1}+\ldots+D^{k} \diamond D^{1} .
\end{aligned}
$$

It is an easy consequence of the axioms that the members of the series are two-sided ideals and the series have the following properties:

$$
\begin{aligned}
& D^{<1>} \supseteq D^{<2>} \supseteq D^{\langle 3\rangle} \supseteq \ldots \supseteq D^{<k>} \supseteq \ldots \\
& D^{\{1\}} \supseteq D^{\{2\}} \supseteq D^{\{3\}} \supseteq \ldots \supseteq D^{\{k\}} \supseteq \ldots \\
& D^{1} \supseteq D^{2} \supseteq D^{3} \supseteq \ldots \supseteq D^{k} \supseteq \ldots
\end{aligned}
$$

The lemma below is on grading properties of the binary operation $\diamond$.

Lemma 1: For any $g, h \in \mathbb{N}$ the following embeddings are valid:

$$
\begin{aligned}
& \text { A. } D^{\langle g\rangle} \diamond D^{\langle h\rangle} \subseteq D^{\langle g+h\rangle}, \\
& \text { B. } D^{\{g\rangle} \diamond D^{\{h\}} \subseteq D^{\{g+h\}} .
\end{aligned}
$$

Proof: The proof we carry out by the induction on $h$ for arbitrary $g$. The verification of the validity of A) for $h=1$ is trivial. Suppose that the embedding is true for $h$ and we prove the statement for $h+1$. It can be easily obtained by using the identities of the diassociative algebra and the following chain of inclusions:

$D^{\langle g\rangle} \diamond D^{\langle h+1\rangle}=D^{\langle g\rangle} \diamond\left(D^{\langle h\rangle} \diamond D\right) \subseteq\left(D^{\langle g\rangle} \diamond D^{h}\right) \diamond D \subseteq D^{\langle g+h\rangle} \diamond D \subseteq D^{\langle g+h+1\rangle}$.

The part B) is showed similarly.

Definition 5: Diassociative algebra $D$ is said to be the right (left) nilpotent if there exists a natural number $k \in \mathbb{N}(p \in \mathbb{N})$ such that $D^{<k>}=0\left(D^{\{p\}}=0\right)$.

Definition 6: Diassociative algebra $D$ is said to be nilpotent if there exists a natural number $s \in \mathbb{N}$ such that $D^{s}=0$.

Lemma 2: For any $g \in \mathbb{N}$ the following equalities hold:

$$
D^{<g>}=D^{\{g\}}=D^{g} .
$$

Proof: We show that $D^{<g>}=D^{g}$, the other cases can be showed similarly. It is obvious that $D^{<g\rangle} \subseteq D^{g}$ for any $g \in \mathbb{N}$. Due to Lemma 1 we have $D^{\langle g\rangle} \diamond D^{\langle h\rangle} \subseteq D^{\langle g+h\rangle}$. It is not difficult to see that 
$D^{<1>}=D^{1}, D^{<2>}=D^{2}$. We make use the mathematical induction method again to show that $D^{g+1} \subset D^{<g+1>}$ assuming that $D^{<g>}=D^{g}$. Indeed,

$$
\begin{aligned}
& D^{g+1}=D^{1} \diamond D^{g}+D^{2} \diamond D^{g-1}+\ldots+D^{g} \diamond D^{1} \\
& \subseteq D^{<1>} \diamond D^{\langle g\rangle}+D^{<2>} \diamond D^{\langle g-1\rangle}+\ldots+D^{\langle g\rangle} \diamond D^{<1>} \\
& \subseteq D^{\langle g+1\rangle} .
\end{aligned}
$$

From this theorem we get the following important corollary.

Corollary 1: The notions of the right nilpotency, left nilpotency and nilpotency of diassociative algebras are equivalent.

Let us now prove the following subsidiary lemma on centralization of monomials in diassociative algebras.

Lemma 3: Let $(D, \dashv, \vdash)$ be a diassociative algebra and $M=x_{1} * x_{2} *$ $\ldots * x_{n}$ be a monomial in $D$, with any parenthesizing, where each $*$ is one of the operations $\dashv, \vdash$.

Then $M$ can be written in the form

$$
y_{-m} \vdash y_{-m+1} \vdash y_{-m+2} \vdash \ldots \vdash y_{-1} \vdash y_{0} \dashv y_{1} \dashv y_{2} \dashv \ldots \dashv y_{k},
$$

where each $y_{r}$ is one of the $x_{\xi}, r=-m,-m+1, \ldots, k$ and $s=1,2, \ldots, n$.

The element $y_{0}$ of the monomial $y_{-m} \vdash y_{-m+1} \vdash y_{-m+2} \vdash \ldots \vdash y_{-1} \vdash y_{0} \dashv y_{1} \dashv y_{2} \dashv \ldots \dashv y_{k}$ is said to be its center. Note that no multipliers from the left and right sides of the center also are possible, i.e., the term is monomial with respect to only one binary operation.

Proof: The proof is carried out by the induction with respect to the number of multi- pliers of monomial. We start with three multipliers case. The following parenthesizes may occur

$$
\left(x_{1} * x_{2}\right) * x_{3}, x_{1} *\left(x_{2} * x_{3}\right),
$$

where $*=\dashv, \vdash$. It is easy to see that diassociative algebra axioms provide the required forms for these monomials. This gives the base of the induction.

Let us now assume that the assertion is true for $n=s$ and prove it for $n=s+1$. Consider $M=x_{1} * x_{2} * \ldots * x_{s+1}$. Then for $M$ we have the following four possibilities:

$$
x_{1} \dashv h, \quad x_{1} \vdash h, \quad h \dashv x_{s+1}, \quad h \vdash x_{s+1},
$$

where $h$ has the required form. In the second and third cases $M$ has the required form. As for the other two external cases we repeatedly make use the associativity property of the products and diassociative algebra axioms to bring them to the required form.

As is observed from the definition of diassociative algebra that it is a combination of two associative structures

$(D, \dashv)$ and $(D, \vdash)$. The following theorem gives an important criterion on verification of nilpotency property of the diassociative algebras. According to that for the verification of the nilpotency of diassociative algebra it suffices to find out one of the associative parts to be nilpotent.

Theorem 3.1: Let $(D, \dashv, \vdash)$ be a finite dimensional diassociative algebra, $A_{1}=(D,-1)$ and $A_{2}=(D, \vdash)$ be corresponding associative algebras. Then the following are equivalent:

i) The diassociative algebra $(D, \dashv, \vdash)$ is nilpotent,

ii) The associative algebra $A_{1}$ is nilpotent,

iii) The associative algebra $A_{2}$ is nilpotent.
Proof: It is obvious that i) follows ii) and iii).

Let us prove that iii) implies ii). Indeed, if the associative algebra $A_{2}=(D, \vdash)$ is nilpotent then there exists $k \in \mathbb{N}$ such that $A_{2}^{k}=0$ (with respect to the right product $\vdash)$. We show that $A_{1}^{k+1}=0$. Let $x \in A_{1}^{k+1}$, i.e.,

$$
x \in \operatorname{Span}_{\mathbb{C}}\left\{x_{1} \dashv x_{2} \dashv \ldots \dashv x_{k+1} \mid x_{i} \in A_{1}\right\} .
$$

Then making repeated use of the associativity of $\dashv$ and axiom 1 in the definition of diassociative algebra we get

$$
x=\left(\ldots\left(\left(x_{1} \dashv x_{2}\right) \dashv x_{3}\right) \dashv \ldots \dashv x_{k+1}\right)=x_{1} \dashv(\underbrace{x_{2} \vdash x_{3} \vdash \ldots \vdash x_{k+1}}_{=0})=x_{1} \dashv 0=0 .
$$

The implication ii) $\Rightarrow$ iii) is proved similarly.

Let show the implication ii) $\Rightarrow$ i). According to the discussion above the statement iii) also holds. Therefore, there exists $k \in \mathbb{N}$ such that $A_{1}^{k}=0$ and $A_{2}^{k+2}=0$. We show that $D^{2 k+2}=\{0\}$. Indeed, since $x \in \operatorname{Span}_{\mathbb{C}}\left\{x_{1} * x_{2} * \ldots * x_{2 k+2} \mid x_{i} \in D\right.$ and $\left.*=-1, \vdash\right\}$ then due to Lemma 3 each of $x_{1} * x_{2} * \ldots * x_{2 k+2}$ is written in the following form:

$$
y_{-m} \vdash y_{-m+1} \vdash y_{-m+2} \vdash \ldots \vdash y_{-1} \vdash y_{0} \dashv y_{1} \dashv y_{2} \dashv \ldots \dashv y_{n},
$$

with $y_{j} \in D$ and $m+n=2 k+2$. In each of these terms the number of multipliers from the left and right sides of the center $y_{0}$ succeeds $k+1$, therefore that part equals zero. This completes the proof.

\section{Example 1:}

a) According to the criterion above among the diassociative algebras in Theorem 2.1 there is only one isomorphism class consisting of entirely nilpotent diassociative algebras: $\operatorname{Dias}_{2}^{3}(\alpha)$.

b) There is only one isomorphism class among three-dimensional diassociative algebras which is nilpotent: Dias ${ }_{3}^{16}$. (see Theorem 2.2).

c) If a diassociative algebra $D$ is nilpotent then its opposite $\left(D,-\dashv^{\prime}, \vdash^{\prime}\right)$ defined by $x \dashv^{\prime} y=y \vdash x$ and $x \vdash^{\prime} y=y \dashv x$ also is nilpotent.

Definition 7: An ideal of diassociative algebra is said to be nilpotent if it is nilpotent as diassociative algebra.

The following fact can be easily proved.

Lemma 4: The sum of two nilpotent ideals of diassociative algebra is nilpotent.

A diassociative algebra has its maximal nilpotent ideal called nilradical.

Similarly to Lie algebras case the concept of solvability for diassociative algebras is defined by using the termination of following recursively defined (derived) series of ideals:

IV. $D^{[1]}=D, D^{[k+1]}=D^{[k]} \diamond D^{[k]}$

However, the following theorem shows that there is no nonnilpotent solvable diassociative algebra.

Theorem 3.2: Let $(D, \dashv, \vdash)$ be finite dimensional diassociative algebra and $A_{1}=(D, \dashv), \quad A_{2}=(D, \vdash)$ be the corresponding associative algebras. Then the following are equivalent:

i) The diassociative algebra $(D, \dashv, \vdash)$ is solvable,

ii) $A_{1}$ is solvable,

iii) $A_{2}$ is solvable.

Proof: The proof of the parts ii) $\Leftrightarrow$ iii), i) $\Rightarrow$ ii) and i) $\Rightarrow$ iii) are obvious. It remains to show the implication ii) $\Rightarrow$ i). Let $A_{1}$ to be solvable, i.e. there exist $k \in \mathbb{N}$ such that $A_{1}^{[k]}=0$. Equivalently, $A_{2}$ also 
is solvable, i.e., there exists $l \in \mathbb{N}$ such that $A_{2}^{[l]}=0$. these mean that any element of

$$
\begin{aligned}
& \operatorname{Span}_{\mathbb{C}}\left\{a_{1} \dashv a_{2} \dashv \ldots \dashv a_{p} \mid a_{i} \in A_{1}, i=1,2, \ldots, p\right\} \text { with } p>2^{k-1} \\
& \text { and } \\
& \operatorname{Span}_{\mathbb{C}}\left\{a_{1} \vdash a_{2} \vdash \ldots \vdash a_{p} \mid a_{i} \in A_{1}, i=1,2, \ldots, p\right\} \text { with } p>2^{l-1}
\end{aligned}
$$

is zero.

We show that there is a natural number $m$ such that $D^{[m]}=\{0\}$. Indeed, let us take $m>\log _{2}\left(2^{k}+2^{l}+1\right)+1$. Then

$$
D^{[m]}=\operatorname{Span}_{\mathbb{C}}\left\{d_{1} * d_{2} * \ldots * d_{2^{m-1}} \mid d_{i} \in D, i=1,2, \ldots, 2^{m-1} \text { and } *=-,, \vdash\right\} .
$$

According to Lemma 3 the element $d_{1} * d_{2} * \ldots * d_{2 m-1}$ can be represented as follows

$$
y_{-s} \vdash y_{-s+1} \vdash y_{-s+2} \vdash \ldots \vdash y_{-1} \vdash y_{0} \dashv y_{1} \dashv y_{2} \dashv \ldots \dashv y_{t},
$$

where $s+t=2^{m-1}+1$. It is clear that in each such term under the condition either the left or right sides of the center is zero. Therefore $D^{[m]}=\{0\}$.

Corollary 2: There is no non-nilpotent solvable diassociative algebra.

The proof is an immediate consequence of the theorem and the fact that there is no non-nilpotent solvable associative algebra.

\section{Classification of Four Dimensional Nilpotent Diassociative Algebras}

In this section we give lists of complex four-dimensional nilpotent diassociative algebras. The idea is as follows. The first part of the diassociative algebra we choose from the list given in [25]. Combining algebra from this list (taking into account the diassociative algebra axioms) with another associative algebra we get a system of equations with respect to the structure constants of the later associative algebra. Solving the system of equations we find all such possible combinations. Then we distinguish non-isomorphic algebras. The next theorem is the classification result of four-dimensional nilpotent associative algebras which we make use in the paper.

Theorem 4.1: Any four-dimensional complex nilpotent associative algebra can be included in one of the following isomorphism classes of algebras:

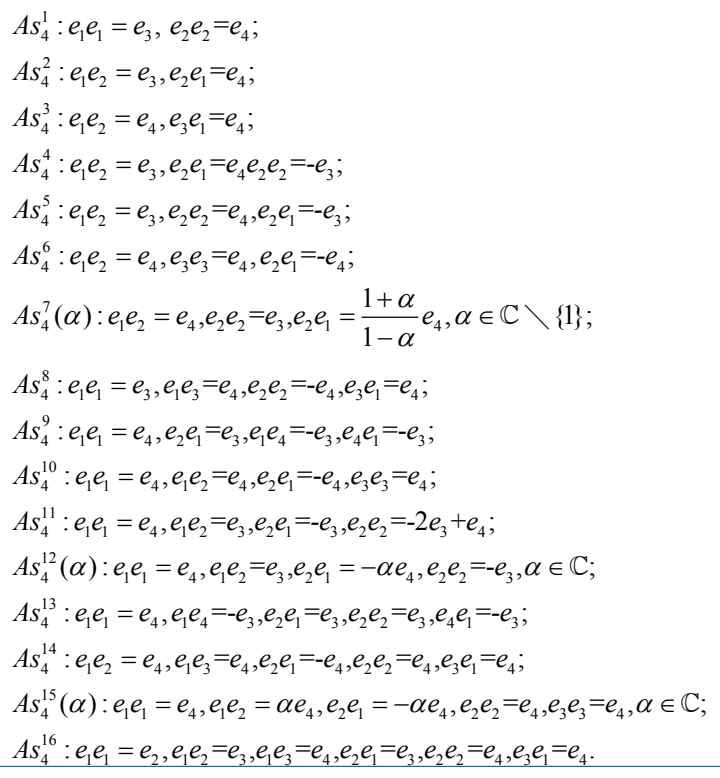

\begin{tabular}{|c|c|c|}
\hline Dias $_{4}^{1}$ : & $\begin{array}{l}e_{1} \dashv e_{1}=e_{3}, \\
e_{1} \vdash e_{1}=a e_{3}+b e_{4}, \\
e_{2} \vdash e_{1}=f e_{3}+g e_{4},\end{array}$ & $\begin{array}{l}e_{2} \dashv e_{2}=e_{4}, \\
e_{1} \vdash e_{2}=c e_{3}+d e_{4}, \\
e_{2} \vdash e_{2}=h e_{3}+k e_{4} ;\end{array}$ \\
\hline $\operatorname{Dias}_{4}^{2}:$ & $\begin{array}{l}e_{1} \dashv e_{2}=e_{3}, \\
e_{1} \vdash e_{1}=a e_{3}+b e_{4}, \\
e_{2} \vdash e_{1}=f e_{3}+g e_{4}\end{array}$ & $\begin{array}{l}e_{2} \dashv e_{1}=e_{4}, \\
e_{1} \vdash e_{2}=c e_{3}+d e_{4}, \\
e_{2} \vdash e_{2}=h e_{3}+k e_{4} ;\end{array}$ \\
\hline $\operatorname{Dias}_{4}^{3}$ : & $\begin{array}{l}e_{1} \dashv e_{2}=e_{4} \\
e_{1} \vdash e_{1}=a e_{4} \\
e_{1} \vdash e_{3}=c e_{4} \\
e_{2} \vdash e_{2}=f e_{4} \\
e_{3} \vdash e_{1}=h e_{4} \\
e_{3} \vdash e_{3}=l e_{4}\end{array}$ & $\begin{array}{l}e_{3} \dashv e_{1}=e_{4} \\
e_{1} \vdash e_{2}=b e_{4} \\
e_{2} \vdash e_{1}=d e_{4} \\
e_{2} \vdash e_{3}=g e_{4} \\
e_{3} \vdash e_{2}=k e_{4}\end{array}$ \\
\hline $\operatorname{Dias}_{4}^{4}:$ & $\begin{array}{l}e_{1} \dashv e_{2}=e_{3}, \\
e_{2} \dashv e_{2}=-e_{3}, \\
e_{1} \vdash e_{2}=c e_{3}+d e_{4}, \\
e_{2} \vdash e_{2}=h e_{3}+k e_{4}\end{array}$ & $\begin{array}{l}e_{2} \dashv e_{1}=e_{4}, \\
e_{1} \vdash e_{1}=a e_{3}+b e_{4}, \\
e_{2} \vdash e_{1}=f e_{3}+g e_{4},\end{array}$ \\
\hline Dias $_{4}^{5}:$ & $\begin{array}{l}e_{1} \dashv e_{2}=e_{3}, \\
e_{2} \dashv e_{2}=e_{4}, \\
e_{1} \vdash e_{2}=c e_{3}+d e_{4}, \\
e_{2} \vdash e_{2}=h e_{3}+k e_{4} ;\end{array}$ & $\begin{array}{l}e_{2} \dashv e_{1}=-e_{3}, \\
e_{1} \vdash e_{1}=a e_{3}+b e_{4}, \\
e_{2} \vdash e_{1}=f e_{3}+g e_{4},\end{array}$ \\
\hline Dias $_{4}^{6}$ : & $\begin{array}{l}e_{1} \dashv e_{2}=e_{4}, \\
e_{3} \dashv e_{3}=e_{4}, \\
e_{1} \vdash e_{2}=b e_{4}, \\
e_{2} \vdash e_{1}=d e_{4}, \\
e_{2} \vdash e_{3}=g e_{4}, \\
e_{3} \vdash e_{2}=k e_{4},\end{array}$ & $\begin{array}{l}e_{2} \dashv e_{1}=-e_{4}, \\
e_{1} \vdash e_{1}=a e_{4}, \\
e_{1} \vdash e_{3}=c e_{4}, \\
e_{2} \vdash e_{2}=f e_{4}, \\
e_{3} \vdash e_{1}=h e_{4}, \\
e_{3} \vdash e_{3}=l e_{4} ;\end{array}$ \\
\hline $\operatorname{Dias}_{4}^{7}:$ & $\begin{array}{l}e_{1} \dashv e_{2}=e_{4}, \\
e_{2} \dashv e_{1}=\frac{1+\alpha}{1-\alpha} e_{4}, \\
e_{1} \vdash e_{2}=c e_{3}+d e_{4}, \\
e_{2} \vdash e_{2}=h e_{3}+k e_{4}\end{array}$ & $\begin{array}{l}e_{2} \dashv e_{2}=e_{3}, \\
e_{1} \vdash e_{1}=a e_{3}+b e_{4}, \\
e_{2} \vdash e_{1}=f e_{3}+g e_{4},\end{array}$ \\
\hline $\operatorname{Dias}_{4}^{8}$ : & $\begin{array}{l}e_{1} \dashv e_{1}=e_{3}, \\
e_{3} \dashv e_{1}=e_{4}, \\
e_{1} \vdash e_{2}=b e_{4}, \\
e_{2} \vdash e_{1}=c e_{4}, \\
e_{3} \vdash e_{1}=e_{4}\end{array}$ & $\begin{array}{l}e_{1} \dashv e_{3}=e_{4}, \\
e_{1} \vdash e_{1}=e_{3}+a e_{4}, \\
e_{1} \vdash e_{3}=e_{4}, \\
e_{2} \vdash e_{2}=d e_{4},\end{array}$ \\
\hline Dias $_{4}^{9}$ : & $\begin{array}{l}e_{1} \dashv e_{1}=e_{4}, \\
e_{2} \dashv e_{1}=e_{3}, \\
e_{1} \vdash e_{1}=a e_{3}+e_{4}, \\
e_{1} \vdash e_{4}=-e_{3}, \\
e_{2} \vdash e_{2}=d e_{3},\end{array}$ & $\begin{array}{l}e_{1} \dashv e_{4}=-e_{3}, \\
e_{4} \dashv e_{1}=-e_{3}, \\
e_{1} \vdash e_{2}=b e_{3}, \\
e_{2} \vdash e_{1}=c e_{3}, \\
e_{4} \vdash e_{1}=-e_{3} ;\end{array}$ \\
\hline $\operatorname{Dias}_{4}^{10}:$ & $\begin{array}{l}e_{1} \dashv e_{1}=e_{4}, \\
e_{2} \dashv e_{1}=-e_{4}, \\
e_{1} \vdash e_{1}=a e_{4}, \\
e_{1} \vdash e_{3}=c e_{4}, \\
e_{2} \vdash e_{2}=f e_{4}, \\
e_{3} \vdash e_{1}=h e_{4}, \\
e_{3} \vdash e_{3}=l e_{4} ;\end{array}$ & $\begin{array}{l}e_{1} \dashv e_{2}=e_{4}, \\
e_{3} \dashv e_{3}=e_{4}, \\
e_{1} \vdash e_{2}=b e_{4}, \\
e_{2} \vdash e_{1}=d e_{4}, \\
e_{2} \vdash e_{3}=g e_{4}, \\
e_{3} \vdash e_{2}=k e_{4},\end{array}$ \\
\hline $\operatorname{Dias}_{4}^{11}$ & $\begin{array}{l}e_{1} \dashv e_{1}=e_{4}, \\
e_{2} \dashv e_{1}=-e_{3}, \\
e_{1} \vdash e_{1}=a e_{3}+b e_{4}, \\
e_{2} \vdash e_{1}=f e_{3}+g e_{4},\end{array}$ & $\begin{array}{l}e_{1} \dashv e_{2}=e_{3}, \\
e_{2} \dashv e_{2}=-2 e_{3}+e_{4}, \\
e_{1} \vdash e_{2}=c e_{3}+d e_{4}, \\
e_{2} \vdash e_{2}=h e_{3}+k e_{4} ;\end{array}$ \\
\hline
\end{tabular}

Now we prove the following theorem which gives all isomorphism classes of four- dimensional complex nilpotent diassociative algebras.

Theorem 4.2: Any four-dimensional complex nilpotent diassociative algebra is isomorphic to one of the following algebras: 


\begin{tabular}{|c|c|c|}
\hline $\operatorname{Dias}_{4}^{12}:$ & $\begin{array}{l}e_{1} \dashv e_{1}=e_{4}, \\
e_{2} \dashv e_{1}=-a e_{4} \\
e_{1} \vdash e_{1}=a e_{3}+b e_{4}, \\
e_{2} \vdash e_{1}=f e_{3}+g e_{4}\end{array}$ & $\begin{array}{l}e_{1} \dashv e_{2}=e_{3}, \\
e_{2} \dashv e_{2}=-e_{3}, \\
e_{1} \vdash e_{2}=c e_{3}+d e_{4}, \\
e_{2} \vdash e_{2}=h e_{3}+k e_{4} ;\end{array}$ \\
\hline $\operatorname{Dias}_{4}^{13}:$ & $\begin{array}{l}e_{1} \dashv e_{1}=e_{4} e_{1} \dashv e_{4}=-e_{3}, \\
e_{2} \dashv e_{2}=e_{3}, \\
e_{1} \vdash e_{1}=a e_{3}+e_{4}, \\
e_{1} \vdash e_{4}=-e_{3}, \\
e_{2} \vdash e_{2}=d e_{3},\end{array}$ & $\begin{array}{l}e_{2} \dashv e_{1}=e_{3}, \\
e_{4} \dashv e_{1}=-e_{3}, \\
e_{1} \vdash e_{2}=b e_{3}, \\
e_{2} \vdash e_{1}=c e_{3}, \\
e_{4} \vdash e_{1}=-e_{3} ;\end{array}$ \\
\hline $\operatorname{Dias}_{4}^{14}:$ & $\begin{array}{l}e_{1} \dashv e_{2}=e_{4}, \\
e_{2} \dashv e_{1}=-e_{4}, \\
e_{3} \dashv e_{1}=e_{4}, \\
e_{1} \vdash e_{2}=b e_{4}, \\
e_{2} \vdash e_{1}=d e_{4}, \\
e_{2} \vdash e_{3}=g e_{4}, \\
e_{3} \vdash e_{2}=k e_{4},\end{array}$ & $\begin{array}{l}e_{1} \dashv e_{3}=e_{4}, \\
e_{2} \dashv e_{2}=e_{4}, \\
e_{1} \vdash e_{1}=a e_{4}, \\
e_{1} \vdash e_{3}=c e_{4}, \\
e_{2} \vdash e_{2}=f e_{4}, \\
e_{3} \vdash e_{1}=h e_{4}, \\
e_{3} \vdash e_{3}=l e_{4} ;\end{array}$ \\
\hline $\operatorname{Dias}_{4}^{15}:$ & $\begin{array}{l}e_{1} \dashv e_{1}=e_{4}, \\
e_{2} \dashv e_{1}=-a e_{4}, \\
e_{3} \dashv e_{3}=e_{4}, \\
e_{1} \vdash e_{2}=b e_{4}, \\
e_{2} \vdash e_{1}=d e_{4}, \\
e_{2} \vdash e_{3}=g e_{4}, \\
e_{3} \vdash e_{2}=k e_{4},\end{array}$ & $\begin{array}{l}e_{1} \dashv e_{2}=a e_{4}, \\
e_{2} \dashv e_{2}=e_{4}, \\
e_{1} \vdash e_{1}=a e_{4}, \\
e_{1} \vdash e_{3}=c e_{4}, \\
e_{2} \vdash e_{2}=f e_{4}, \\
e_{3} \vdash e_{1}=h e_{4}, \\
e_{3} \vdash e_{3}=l e_{4} ;\end{array}$ \\
\hline $\operatorname{Dias}_{4}^{16}:$ & $\begin{array}{l}e_{1} \dashv e_{1}=e_{2}, \\
e_{1} \dashv e_{3}=e_{4}, \\
e_{2} \dashv e_{2}=e_{4}, \\
e_{1} \vdash e_{1}=e_{2}+a e_{4}, \\
e_{1} \vdash e_{3}=e_{4}, \\
e_{2} \vdash e_{2}=e_{4},\end{array}$ & $\begin{array}{l}e_{1} \dashv e_{2}=e_{3}, \\
e_{2} \dashv e_{1}=e_{3}, \\
e_{3} \dashv e_{1}=e_{4}, \\
e_{1} \vdash e_{2}=e_{3}, \\
e_{2} \vdash e_{1}=e_{3}, \\
e_{3} \vdash e_{1}=e_{4},\end{array}$ \\
\hline
\end{tabular}

Proof: We provide the proof only for one case to illustrate the approach used, the other cases can be carried out similarly by a minor changing.

Let the associative part $A_{1}=(D, \dashv)$ of $\mathrm{D}$ be the algebra from $A s_{4}^{1}$ Theorem 4.1, with the following table of multiplication:

$$
e_{1} \dashv e_{1}=e_{3}, e_{2} \dashv e_{2}=e_{4}
$$

The second part $A_{2}=(D, \vdash)$ of $D$ we define by unknown structure constants $\alpha_{i}, \beta_{i}, \gamma_{i}$ and $\delta_{i}(i=1,2, \ldots, 16)$ as follows:

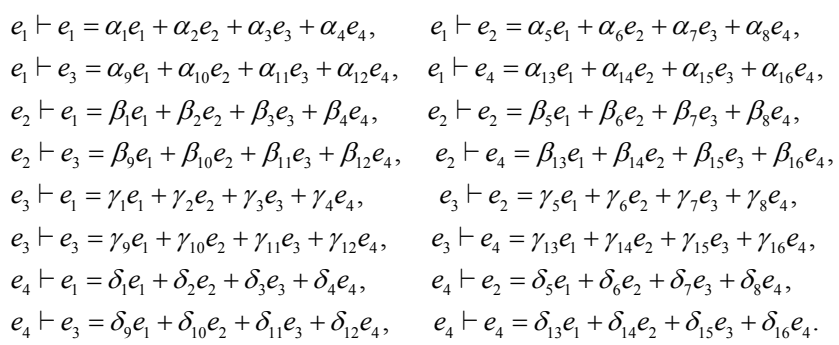

Combining $A_{1}, A_{2}$ and verifying the diassociative algebra axioms we get a system of equations with respect to $\alpha i, \beta_{i}, \gamma_{i}$ and $\delta_{i}$. Solving the system of equations we obtain:

$\alpha_{1}=0, \alpha_{2}=0, \alpha_{5}=0, \alpha_{6}=0, \alpha_{9}=0, \alpha_{10}=0$,

$$
\begin{aligned}
& \alpha_{11}=0, \alpha_{12}=0, \alpha_{13}=0, \alpha_{14}=0, \alpha_{15}=0, \alpha_{16}=0, \\
& \beta_{1}=0, \beta_{2}=0, \beta_{5}=0, \beta_{6}=0, \beta_{9}=0, \beta_{10}=0, \\
& \beta_{11}=0, \beta_{12}=0, \beta_{13}=0, \beta_{14}=0, \beta_{15}=0, \beta_{16}=0, \gamma_{i}=0, \delta_{i}=0
\end{aligned}
$$

where $1 \leq i \leq 16$, and $\alpha_{3}, \alpha_{4}, \alpha_{7}, \alpha_{8}, \beta_{3}, \beta_{4}, \beta_{7}, \beta_{8} \in \mathbb{C}$.

Denoting the structure constants $\alpha_{3}, \alpha_{4}, \alpha_{7}, \alpha_{8}, \beta_{3}, \beta_{4}, \beta_{7}, \beta_{8}$ by $\alpha_{3}=$ $a, \alpha_{4}=b, \alpha_{7}=c, \alpha_{8}=d, \beta_{3}=f, \beta_{4}=g, \beta_{7}=h, \beta_{8}=k$, we get the family of algebras:

$$
\begin{aligned}
\operatorname{Dias}_{4}^{1}: & e_{1} \dashv e_{1}=e_{3}, e_{2} \dashv e_{2}=e_{4}, e_{1} \vdash e_{1}=a e_{3}+b e_{4}, \\
& e_{1} \vdash e_{2}=c e_{3}+d e_{4}, e_{2} \vdash e_{1}=f e_{3}+g e_{4}, \\
& e_{2} \vdash e_{2}=h e_{3}+k e_{4} .
\end{aligned}
$$

The results of the other combinations are given in the following Table 1.

Note that there are isomorphic algebras in each Dias $_{4}^{i}$, where $i \neq 4,12,13,16$. The conditions for algebras to be isomorphic inside the classes are given as follows.

$$
\begin{aligned}
& \operatorname{Dias}_{4}^{1}(a, b, c, d, f, g, h, k) \cong \operatorname{Dias}_{4}^{1}\left(a, \frac{1}{\mu^{2}} b, \mu c, \frac{1}{\mu} d, \mu f, \frac{1}{\mu} g, \mu^{2} h, k\right), \mu \neq 0 ; \\
& \operatorname{Dias}_{4}^{2}(a, b, c, d, f, g, h, k) \cong \operatorname{Dias}_{4}^{2}\left(\mu a, \mu b, \mu c, d, f, g, \frac{1}{\mu} h, \frac{1}{\mu} k\right), \mu \neq 0 ; \\
& \operatorname{Dias}_{4}^{3}(a, b, c, d, f, g, h, k, l) \cong \operatorname{Dias}_{4}^{3}\left(\frac{1}{\mu} a, b, c, d, \mu f, \mu g, h, \mu k, \mu l\right), \mu \neq 0 ; \\
& \operatorname{Dias}_{4}^{5}(a, b, c, d, f, g, h, k) \cong \operatorname{Dias}_{4}^{5}\left(\mu a, \mu^{2} b, c, \mu d, f, \mu g, \frac{1}{\mu} h, k\right), \mu \neq 0 ; \\
& \operatorname{Dias}_{4}^{6}(a, b, c, d, f, g, h, k) \cong \operatorname{Dias}_{4}^{6}\left(\mu^{2} a, b, \mu c, d, \frac{1}{\mu^{2}} f, \frac{1}{\mu} g, \mu h, \frac{1}{\mu} k, l\right), \mu \neq 0 ; \\
& \operatorname{Dias}_{4}^{7}(\beta, a, b, c, d, f, g, h, k) \cong \operatorname{Dias}_{4}^{7}\left(\beta, \mu 2 a, \mu b, \mu c, d, \mu f, g, h, \frac{1}{\mu} k\right), \mu \neq 0 ; \\
& \operatorname{Dias}_{4}^{8}(a, b, c, d) \cong D i a s_{4}^{8}\left(\mu^{2} a, \mu b, \mu c, d\right), \mu \neq 0 ; \\
& \operatorname{Dias}_{4}^{9}(a, b, c, d) \cong \operatorname{Dias}{ }_{4}^{9}\left(\mu a, b, c, \frac{1}{\mu} d\right), \mu \neq 0 ; \\
& \operatorname{Dias}_{4}^{10}(a, b, c, d, f, g, h, k, l) \cong \operatorname{Dias}_{4}^{10}\left(a, b, \frac{1}{\mu} c, d, f, \mu g, \mu h, \mu k, l\right), \mu \neq 0 ; \\
& \operatorname{Dias}_{4}^{11}(a, b, c, d, f, g, h, k) \cong \operatorname{Dias}_{4}^{11}\left(\mu a, b, c, \frac{1}{\mu} d, f, \mu g, h, k\right), \mu \neq 0 ; \\
& \operatorname{Dias}_{4}^{14}(a, b, c, d, f, g, h, k, l) \cong \operatorname{Dias}_{4}^{14}\left(a, b, \mu c, d, f, \mu g, \mu h, \mu k, \mu^{2} l\right), \mu \neq 0 ; \\
& \operatorname{Dias}_{4}^{15}(a, b, c, d, f, g, h, k, l) \cong \operatorname{Dias}_{4}^{15}(a, \mu b,-\mu c, \mu d, f,-g,-\mu h,-k, l), \mu \neq 0 .
\end{aligned}
$$

The verification of the diassociativity axioms and isomorphisms between the algebras found we used two computer programs in Maple which are available from the authors.

\section{Acknowledgment}

The second author was supported by the research grant 01-02-14-1591FR MOHE, Malaysia. The research of the third author was supported by FRGS/ FASA1-2007/Sains Tulen/UPM/ 297 and Short Term Research Grant UniKL IRPS/str11061. The authors gratefully acknowledge the referee for the valuable comments and Sh.Rakhimov for the discussions on computational part of the paper.

\section{References}

1. Ayupov Sh A, Omirov BA (1998) On Leibniz algebras. Algebra and operators theory, Kluwer Academic Publishers, Netherlands.

2. Casas JM, Insua MA, Ladra MA, Ladra S (2012) An algorithm for the classification of 3-dimensional complex Leibniz algebras. Linear Algebra and App 436: 3747-3756.

3. Albeverio S, Omirov BA, Rakhimov IS (2005) Varieties of nilpotent complex Leibniz algebras dimensions less than five, Comm. in Algebra 33: 1575-1585. 
Citation: Basri W, Rakhimov IS, Rikhsiboev IM (2015) Four-Dimensional Nilpotent Diassociative Algebras. J Generalized Lie Theory Appl 9: 218. doi: 10.4172/1736-4337.1000218

Page 7 of 7

4. Loday JL, Frabetti A, Chapoton F, Goichot F (2001) Dialgebras and Related Operads, Lecture Notes in Math., Springer, Berlin.

5. Gabriel R (1975) Finite representation type is open. Lecture Notes in Math. 488: 132-155.

6. Mazolla G (1979) The algebraic and geometric classification associative algebras of dimension five. Manuscripta Math 27: 81-101.

7. Goze M, Khakimjonov Yu (1996) Nilpotent Lie algebras. Kluwer Academic Publishers, Netherlands.

8. Jacobson N (1962) Lie algebras. Interscience Tracts in Pure and Applied Mathematics, New York.

9. Basri W, Rikhsiboev IM (2007) On low dimensional diassociative algebras. Proceedings of Third Conference on Research and Education in Mathematics (ICREM-3), UPM, Malaysia.

10. Rikhsiboev IM, Rakhimov IS, Basri W (2010) Classification of 3-dimensional complex diassociative algebras. Malaysian Journal of Mathematical Sciences 4: 241-254.

11. Loday JL (1993) Une version non commutative des algebras de Lie: les algebras de Leibniz. Enseign Math 39: 269-293.

12. Gonzalez CM (2013) Associative dialgebras from structural viewpoint. Comm in Algebra 41: 1903-1912.

13. Lin L, Zhang Y (2010) F [x, y] as a dialgebra and a Leibniz algebra. Comm. in Algebra 38: 3417-3447.

14. Velasquez R, Felipe R (2009) Split dialgebras, split quasi-Jordan algebras and regular elements. Journal Algebra Appl 8: 191-218.
15. Ebrahimi-Fard K, Guo L, Rota-Baxter algebras and dendriform algebras. J Pure and Appl Alg 212: 320-339.

16. Felipe $\mathrm{R}$ (2013) Dendriform algebras and Rota-Baxter operators revisited in several directions, Communicaciotns in CIMAT.

17. Goichot F (2001) Decomposition of dendriform homology for Zinbiel algebras.

18. Dzhumadildaev AS, Tulenbaev KM (2005) Nilpotency of Zinbiel algebras. J Dyn. Control Syst 11: 195-213.

19. Omirov BA (2002) Classification of two dimensional complex Zinbiel algebras Uzbek Math. Jour 2: 55-59.

20. Canete EM, Khudoyberdiyev AKH (2013) The classification of 4-dimensional Leibniz algebras. Linear Algebra Appl 439: 273-288.

21. Casas JM, Ladra M, Omirov BA, Karimjanov IA (2013) Classification of solvable Leibniz algebras with naturally graded filiform nilradical. Linear Algebra and Appl 438: 2973-3000.

22. Abdulkareem AO, Rakhimov IS, Said Husain SK (2015) Isomorphism classes and invariants of low-dimensional filiform Leibniz algebras, Linear and Multilinear algebra 63: 2254-2274.

23. Omirov BA, Rakhimov (2009) IS On Lie-like filiform Leibniz algebras. Bulletin of the Australian Mathematical Society 79: 391-404.

24. Rakhimov IS, Said Husain SK (2011) On isomorphism classes and invariants of a subclass of low dimensional complex filiform Leibniz algebras. Linear and Multilinear algebra 59: 205-220.

25. Rakhimov IS, Rikhsiboev IM, Basri W (2009) Complete lists of low dimensiona complex associative algebras. 